\title{
LHC Higgs Cross-Section Working Group report
}

\author{
Nikolaos Rompotis* \\ University of Liverpool \\ E-mail: nikolaos.rompotisecern.ch
}

The LHC Higgs Cross-Section Working Group is a cooperation between experimentalists from the general purpose LHC experiments and phenomenologists that aims at harmonizing the procedures and the theory inputs used in Higgs boson searches and measurements. This talk is an overview of the structure and the activities of the working group in the past two years with emphasis on the beyond-Standard-Model Higgs boson topics.

Prospects for Charged Higgs Discovery at Colliders

3-6 October 2016

Uppsala, Sweden

${ }^{*}$ Speaker. 


\section{Introduction}

The discovery of a Higgs boson $[1,2]$ at the Large Hadron Collider (LHC) general purpose experiments, ATLAS [3] and CMS [4], has been a joint venture comprising a large number of experts on detectors, accelerators, data-analysis and phenomenology. This document will discuss some of the aspects of the fruitful dialogue between phenomenology and experiment that has been conducted within the LHC Higgs Cross-Section Working Group (LHC HXSWG).

The LHC HXSWG [5] was formed in 2010 as a cooperation among volunteers from ATLAS and CMS experiments and the phenomenology community. The target of the group was to provide state-of-the-art predictions for production cross-sections and branching ratios relevant to Higgs boson searches and to harmonize the theory inputs for ATLAS and CMS analyses, such that their results are (more) comparable. This task includes predictions for Standard Model (SM) Higgs boson production and decay, but also for Higgs bosons in well-studied beyond SM theories, like the Minimal Supersymmetric SM (MSSM) (see e.g. [6]). After the Higgs boson discovery, the group expanded to include Higgs boson property measurement strategy and interpretation.

In this document, a summary of the group structure is given, as it was between 2014 and 2016, along with a short description of the studies performed during the same period.

\section{Structure and documentation of the results}

The LHC HXSWG since October 2014 is coordinated by a Steering Committee, which is comprised by two members representing the ATLAS collaboration, two members representing the CMS collaboration, and two phenomenologists. The phenomenologists are proposed by the Theory Advisory Committee, which is comprised of eight senior theorists. The work is divided among three working groups, each of which is coordinated by two ATLAS representatives, two CMS representatives and two phenomenology conveners.

The three working groups of the LHC HXSWG focus on the following: Higgs boson production cross-sections and decay branching ratios (Working group 1 or "WG1"); Higgs boson properties (Working group 2 or "WG2"); beyond SM Higgs bosons (Working group 3 or "WG3"). Each of the three working groups contains several task-forces or sub-groups that focus on specific topics, e.g. exotic Higgs boson decays. All the details, including the structure, the contact people, as well physics results, can be found in the LHC HXSWG twiki [5]. Participation to the activities is open to all members of the community and instructions on how to subscribe to the mailing list and how to further contribute are included in the previously mentioned twiki.

The mandate of the current coordination, as it was previously described, finishes in October 2016. After, that the group structure will be re-examined.

The LHC HXSWG has been documenting since its foundation the recommendations and the provided cross-sections and branching ratios in CERN "yellow reports". These reports were also submitted to the arXiv pre-print server, where they are freely available. Three such yellow reports have been available so far [7-9] with the first one released in 2011 and the most recent in 2013. Altogether, they have collected more than 2000 citations to-date.

The work performed after the last yellow Report was released is documented in the 4th yellow report (YR4) [10]. Due to the fact that some of the procedures had to be cited in publications by 
the experimental collaborations before the YR4 release, the group provided interim reports and internal notes, which are all available on the CERN Document Server. ${ }^{1}$

\section{SM Higgs boson physics}

This section is only a short summary of the activities of the group related to SM Higgs boson physics. These activities comprise most of the group activity and fall under the umbrellas of WG1 and WG2.

The major update in the production cross section calculation is the calculation of gluon-fusion Higgs boson production cross section in $\mathrm{N}^{3} \mathrm{LO}$ precision in QCD. For the branching ratios there is no significant change compared to the previous reports, apart from the addition of recommendations for rare Higgs boson decays, which are also mentioned in the next session.

The measurement of the Higgs boson properties has been done so far using mostly the " $\kappa$ framework" [9]. The $\kappa$ coefficients are related to the Higgs boson couplings and try to capture deviations by taking into account only overall rates. This approach is adequate as long as the statistical power of the data is small. However, as soon as sensitivity to differential distributions is attained, a more comprehensive framework needs to be put into place. Simplified template cross sections and pseudo-observables are two complementary approaches that have been studied in YR4. The results are interpreted in the context of an effective field theory.

\section{Beyond SM Higgs physics}

The main part of this talk is a discussion of the LHC HXSWG results on beyond SM Higgs bosons. This work is mostly performed in WG3. There are several task forces, the results of which are summarised in the following.

\subsection{Neutral MSSM Higgs bosons}

The main target of this subgroup is to provide recommendations for neutral MSSM Higgs boson searches. The main output has been the calculation of production cross sections for gluonfusion and b-associated production for MSSM Higgs bosons in various benchmark scenarios. ${ }^{2}$ Branching ratios are also provided in cooperation with the Branching Ratio subgroup of WG1.

The legacy $m_{h}^{\max }$ scenario $[12,13]$ has been used since the very beginning of the LHC data taking by both ATLAS and CMS collaborations (see e.g. Fig 1 (a)). This scenario was originally intended for LEP searches and assumes a low supersymmetry breaking scale (1 TeV) and large stop mixing, such that the mass of the lightest CP-even Higgs, $m_{h}$, attains its maximum value, which is

\footnotetext{
${ }^{1}$ https: //cds.cern.ch/collection/LHC20Higgs $\div 20$ Cross $\% 20$ Section $\div 20$ Working 20 Group

${ }^{2}$ The term "benchmark scenario" is used here to describe a particular part of the parameter space of a model, chosen by fixing some of the model parameters and leaving a few others (usually two) free. For example, the MSSM Higgs boson benchmark scenarios discussed in this section have as free parameters the mass of the CP-odd Higgs boson, $m_{A}$, and the ratio of the vacuum expectation values of the two Higgs doublets, $\tan \beta$. The choice of the values of the parameters that are fixed is done such that particular features of the model are probed. For instance, in the $\tau$-phobic scenario [11], the choice is such that the couplings to down-type farmings of the lightest CP-even Higgs boson of the model are suppressed.
} 
$m_{h} \sim 130 \mathrm{GeV}$. After the discovery of a Higgs boson in 2012, the scenarios were updated to adjust compatibility of the predicted mass of the lighter (or heavier) CP-even Higgs boson of the MSSM to the observed value. This is the idea behind $m_{h}^{\bmod +}, m_{h}^{\bmod -}$, light stop, and other scenarios that the interested reader may find in Ref. [9].

These scenarios have difficulty in attaining $m_{h} \sim 125 \mathrm{GeV}$ at low $\tan \beta$ values because they still require a supersymmetry breaking scale of $1 \mathrm{TeV}$. By increasing this scale one can go to lower $\tan \beta$ values. A heuristic approach is done in the "hMSSM" scenario, in which all the superpartners are assumed to be very heavy and only the Higgs sector is below $1 \mathrm{TeV}$. The predictions are then based on lower order MSSM formulas and are valid under certain assumptions. In a complementary approach, the "low-tanb-high" scenario, explicit choices are made for the soft supersymmetry breaking parameters, which are tuned, such that for part of the low $\tan \beta$ region $m_{h} \sim 125 \mathrm{GeV}$. Finally, an effective field theory approach is under study in which the supersymmetry breaking parameters are set to a high energy scale and then the normalisation group evolution is followed to the LHC scale to make predictions about the MSSM Higgs boson observables. Recommended values for this approach are expected to become available at the beginning of next year.

The subgroup has also studied the Higgs boson transverse momentum in gluon-fusion production mode and has provided recommendations for the generation of Monte-Carlo samples. More information can be found in YR4.

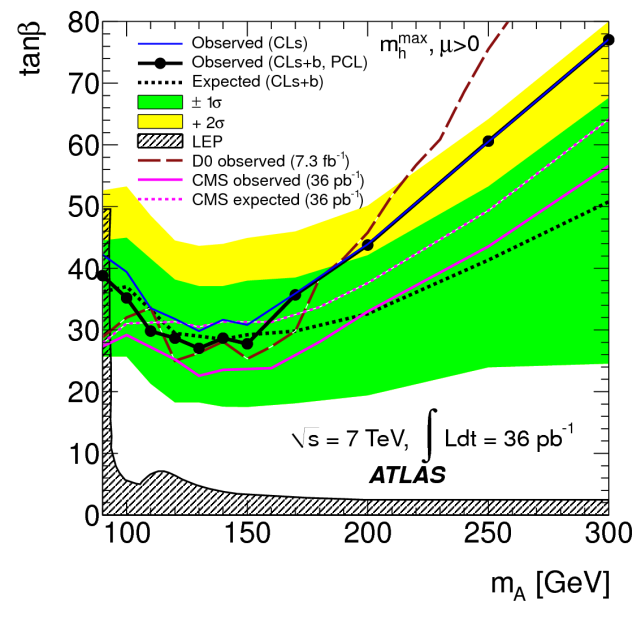

(a)

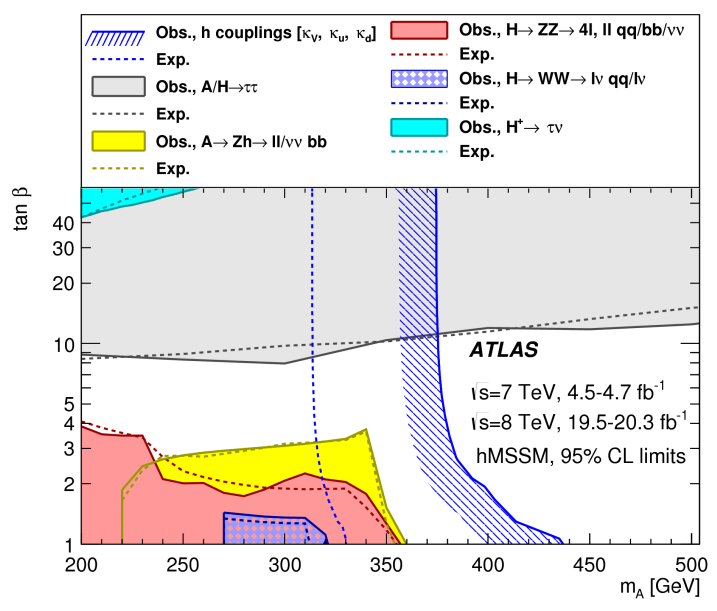

(b)

Figure 1: Examples from the ATLAS collaboration of search and measurement interpretation using MSSM benchmark scenarios provided by the LHC HXSWG. In both cases the results are interpreted in the plane defined by the mass of the MSSM CP-odd Higgs boson, $m_{A}$, and the ratio of the vacuum expectation values of the two Higgs doublets, $\tan \beta$. (a) One of the first LHC MSSM Higgs bosons searches with the first $7 \mathrm{TeV}$ data from Ref. [14] interpreted in the $m_{h}^{\max }$ scenario. (b) Overlay of the constrains from Higgs boson measurements and the searches for additional Higgs bosons interpreted in the "hMSSM" scenario from Ref. [15].

The main client analysis for the results of this subgroup has been the search for a heavy Higgs boson decaying to $\tau \tau$ pairs. However, Higgs boson searches, as well as Higgs boson property 
measurements have been used as well, see e.g. Fig 1 (b).

\subsection{Charged Higgs bosons}

The work of this subgroup focuses on providing recommendations for charged Higgs boson searches. Its main output has been production cross sections for charged Higgs bosons in the various MSSM scenarios that have been used in the neutral MSSM Higgs boson searches.

The subgroup has also provided recommendations about the Monte-Carlo sample generation for charged Higgs boson searches at the LHC. Detailed studies of the differential distributions of charged Higgs bosons produced in association with top and bottom quarks are reported in YR4.

An important topic that is not covered in the last report of the group is the treatment of the "intermediate" charged Higgs boson mass region (see also Ref. [16]). This refers to charged Higgs boson masses that are around the top-quark mass, $m_{\text {top }}$. The difficulty here is that in this region two different production mechanisms interfere with each other. At low charged Higgs boson masses, i.e. below $m_{\text {top }}$, the charged Higgs boson is mostly produced in top-quark decays. However, for masses above $m_{\text {top }}$ it is directly produced in association with top and bottom quarks. The interference of the two production modes was only available at leading order (LO) using e.g. the Madgraph5_aMC@NLO [17] event generator. Members of the group have now performed the first next-to-LO calculation of the inclusive cross section [18] and the resulting cross section grids are available in the LHC HXSWG twiki page. ${ }^{3}$ There are still no available NLO differential distributions and, hence, the current recommendation is to rescale the LO distributions.

The main client analysis for the results of this subgroup has been the search for a charged Higgs boson in the $\tau \nu$ channel, see e.g. Ref. [19,20]. This has recently expanded now to include the $H^{+} \rightarrow t b$ search [21].

\subsection{Extended scalar sector}

The topic of interest of this subgroup is the extensions of the Higgs sector with a scalar singlet, doublet and triplet of fields.

For the two-Higgs-Doublet-Model (2HDM) (see e.g. [22]) there have been already LHC HXSWG recommendations on the calculation of production cross sections since 2012 [23]. Since then, there has been an effort to do a systematic survey of possible benchmark signatures. The results are tabulated with example production cross sections and branching ratios.

A similar procedure has been followed for singlet extensions. The possibility of a real or complex singlet of fields in addition to the SM Higgs doublet was considered. Recommendations on the calculation of cross sections and branching ratios have been provided and a survey of benchmarks, in the same lines as in the 2HDM, has been performed.

The Georgi-Machacek (GM) model [24] has been examined in the case of extensions of the SM with triplet scalar fields. In the GM model, two triplets of fields are added, in a configuration such that at lowest order $\rho \equiv m_{W}^{2} /\left(m_{Z}^{2} \cos ^{2} \theta_{W}\right)=1$. The Higgs fields of the GM model are organised in two singlets, one triplet and one quadruplet under custodial SU(2) symmetry. ${ }^{4}$ This results

\footnotetext{
${ }^{3}$ https://twiki.cern.ch/twiki/bin/view/LHCPhysics/LHCHXSWGMSSMCharged

${ }^{4}$ The Higgs potential has a global $\mathrm{SU}(2)_{\mathrm{L}} \times \mathrm{SU}(2)_{\mathrm{R}}$ symmetry, which after electroweak symmetry breaking is reduced to $\mathrm{SU}(2)_{\mathrm{V}}$. This remaining symmetry is referred to in the literature as "custodial symmetry" (see also Ref. [25]).
} 
in 10 Higgs bosons after electroweak symmetry breaking. The quadruplet states for most of the parameter space are fermiophobic. They are produced via weak vector boson fusion (VBF) and decay to vector bosons. In Ref. [26] recommendations for the VBF production cross sections and the branching ratios for the quadruplet states are provided. These results have already been used by ATLAS and CMS experiments (see Fig. 2 and Refs. [27, 28]).

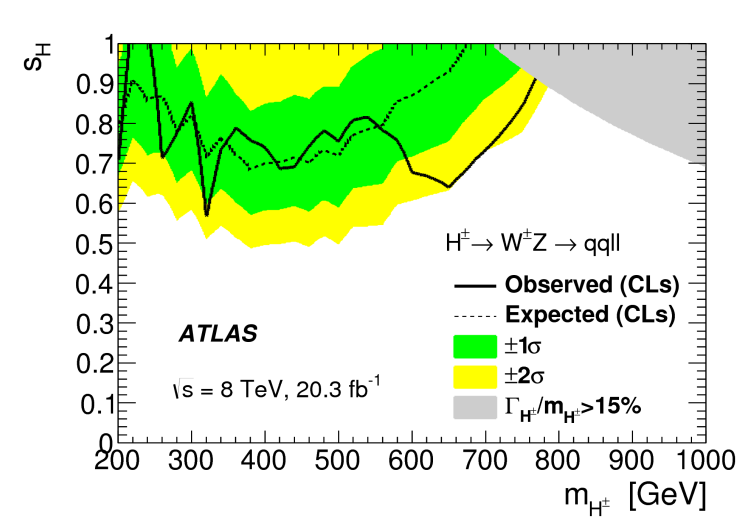

(a)

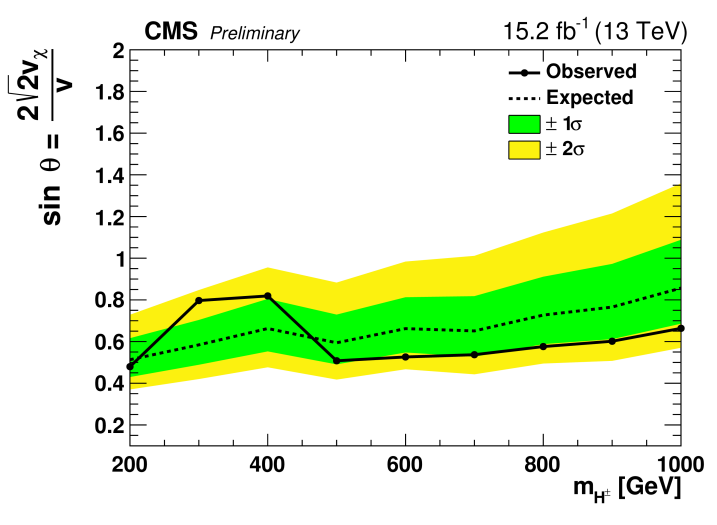

(b)

Figure 2: Examples of the interpretation of the search for a VBF produced charged Higgs boson, $H^{ \pm}$, decaying to $W Z$ in the GM model using the results from LHC HXSWG appearing in Ref. [26]. In both cases the constraints are given on the plane defined by the quadruplet mass parameter, $m_{H \pm}$, and the vacuum expectation value of the triplet, $v_{\chi}$, which is used as $\mathrm{S}_{H} \equiv \sin \theta \equiv 2 \sqrt{2} v_{\chi} / v$, where $v$ is the vacuum expectation value of the SM Higgs doublet. (a) ATLAS result from Ref. [27]. (b) CMS result from Ref. [28].

\subsection{Other topics}

The study of exotic or rare decays of the already discovered Higgs boson has attracted a lot of interest both within the experiments and the theory community, and has led to the formation of the exotic Higgs boson decays subgroup. This subgroup is studying rare SM Higgs boson decays (e.g. see Tab. 1), as well as exotic decays, like Higgs boson to long-lived particles, flavour violating decays (e.g. $h \rightarrow \tau \mu$ ), etc.

The next-to-Minimal Supersymmetric SM (NMSSM) (see e.g. Ref. [29]) is the MSSM with an additional Higgs singlet and a singlino Majorana fermion. The phenomenology becomes much more complicated than the MSSM. In YR4 there has been an effort to survey the available tools for the calculation of the particle spectrum and the production cross sections as well as to look for benchmark scenarios suitable for the LHC searches.

Finally, the $b$-associated Higgs boson production, which is significant in parts of the parameter space of many beyond SM scenarios, like the MSSM, has been studied in detail. Differential distributions and matching between the four-flavour and the five-flavour scheme calculations are all topics discussed in YR4. 


\begin{tabular}{|c|ccc|}
\hline & \multicolumn{3}{|c|}{ Branching fraction $10^{-6}$} \\
& NRQCD & LCDA LO & LCDA NLO \\
\hline $\mathrm{BR}(h \rightarrow \rho \gamma)$ & - & $19.0 \pm 1.5$ & $16.8 \pm 0.8$ \\
$\mathrm{BR}(h \rightarrow \omega \gamma)$ & - & $1.60 \pm 0.17$ & $1.48 \pm 0.08$ \\
$\mathrm{BR}(h \rightarrow \phi \gamma)$ & - & $3.00 \pm 0.13$ & $2.31 \pm 0.11$ \\
$\mathrm{BR}(h \rightarrow J / \psi \gamma)$ & - & $2.79_{-0.15}^{+0.16}$ & $2.95 \pm 0.17$ \\
$\mathrm{BR}(h \rightarrow \Upsilon(1 S) \gamma)$ & $\left(0.61_{-0.61}^{+1.74}\right) \times 10^{-3}$ & - & $\left(4.61_{-1.23}^{+1.76}\right) \times 10^{-3}$ \\
$\mathrm{BR}(h \rightarrow \Upsilon(2 S) \gamma)$ & $\left(2.02_{-1.28}^{+1.86}\right) \times 10^{-3}$ & - & $\left(2.34_{-1.00}^{+0.76}\right) \times 10^{-3}$ \\
$\mathrm{BR}(h \rightarrow \Upsilon(3 S) \gamma)$ & $\left(2.44_{-1.30}^{+1.75}\right) \times 10^{-3}$ & - & $\left(2.13_{-0.76}^{+1.13}\right) \times 10^{-3}$ \\
\hline
\end{tabular}

Table 1: Example of tabulated theoretical prediction for rare SM Higgs boson decays from YR4. For more details, the explanation of the terms and more references, see Ref. [10].

\section{Conclusions}

The LHC HXSWG is a cooperation between ATLAS and CMS experimentalists and phenomenologists that provides theory input for the experimental searches and measurements that are related to Higgs boson physics in the SM and beyond. The group documents its results in CERN yellow reports. There are four such reports, with the fourth one just becoming available. All the LHC SM Higgs boson results, including both searches and measurements, have used input from those reports. The same is true for the majority of the LHC beyond SM Higgs boson search results.

\section{Acknowledgements}

I thank the organisers of Charged 2016 conference for the invitation and the organization of a productive conference in a pleasant atmosphere. I also wish to thank my LHC HXSWG colleagues for the very fruitful collaboration for the past 4 years and in particular Rui Santos, Sven Heinemeyer, Maggie Mühlleitner, and Howard Haber. This work would not have been performed without the support of my mentor and collaborator Anna Goussiou and the University of Washington, Seattle and National Science Foundation grant PHY-1509257. I would also like to acknowledge the support from the University of Liverpool Physics department, which funded my trip to Uppsala. Finally, I wish to thank Spyridon Argyropoulos for reading and commenting on the manuscript.

\section{References}

[1] ATLAS Collaboration, Phys. Lett. B 716 (2012) 1 [arXiv:1207.7214 [hep-ex]].

[2] CMS Collaboration, Phys. Lett. B 716 (2012) 30 [arXiv:1207.7235 [hep-ex]].

[3] ATLAS Collaboration, JINST 3 (2008) S08003.

[4] CMS Collaboration, JINST 3 (2008) S08004.

[5] https://twiki.cern.ch/twiki/bin/view/LHCPhysics/LHCHXSWG

[6] A. Djouadi, Phys. Rept. 459 (2008) 1 [hep-ph/0503173]. 
[7] S. Dittmaier et al. [LHC Higgs Cross Section Working Group Collaboration], arXiv:1101.0593 [hep-ph].

[8] S. Dittmaier et al., arXiv:1201.3084 [hep-ph].

[9] S. Heinemeyer et al. [LHC Higgs Cross Section Working Group Collaboration], arXiv:1307.1347 [hep-ph].

[10] D. de Florian et al. [LHC Higgs Cross Section Working Group Collaboration], arXiv:1610.07922 [hep-ph].

[11] M. Carena, S. Heinemeyer, O. Stål, C. E. M. Wagner and G. Weiglein, Eur. Phys. J. C 73 (2013) no.9, 2552 [arXiv:1302.7033 [hep-ph]].

[12] S. Heinemeyer, W. Hollik and G. Weiglein, JHEP 0006 (2000) 009 [hep-ph/9909540].

[13] M. Carena, S. Heinemeyer, C. E. M. Wagner and G. Weiglein, Eur. Phys. J. C 26 (2003) 601 [hep-ph/0202167].

[14] ATLAS Collaboration, Phys. Lett. B 705 (2011) 174 [arXiv:1107.5003 [hep-ex]].

[15] ATLAS Collaboration, JHEP 1511 (2015) 206 [arXiv:1509.00672 [hep-ex]].

[16] M. Flechl, PoS(CHARGED2016)013.

[17] J. Alwall et al., JHEP 1407 (2014) 079 [arXiv:1405.0301 [hep-ph]].

[18] C. Degrande, R. Frederix, V. Hirschi, M. Ubiali, M. Wiesemann and M. Zaro, arXiv:1607.05291 [hep-ph].

[19] ATLAS Collaboration, Phys. Lett. B 759 (2016) 555 [arXiv:1603.09203 [hep-ex]].

[20] CMS Collaboration, JHEP 1511 (2015) 018 [arXiv:1508.07774 [hep-ex]].

[21] ATLAS Collaboration, ATLAS-CONF-2016-089 (2016).

[22] G. C. Branco, P. M. Ferreira, L. Lavoura, M. N. Rebelo, M. Sher and J. P. Silva, Phys. Rept. 516 (2012) 1 [arXiv:1106.0034 [hep-ph]].

[23] R. Harlander, M. Mühlleitner, J. Rathsman, M. Spira and O. Stål, arXiv:1312.5571 [hep-ph].

[24] H. Georgi and M. Machacek, Nucl. Phys. B 262 (1985) 463.

[25] J. F. Gunion, H. E. Haber, G. L. Kane and S. Dawson, Front. Phys. 80 (2000) 1.

[26] M. Zaro and H. Logan, LHCHXSWG-2015-001 (2015).

[27] ATLAS Collaboration, Phys. Rev. Lett. 114 (2015) no.23, 231801 [arXiv:1503.04233 [hep-ex]].

[28] CMS Collaboration, CMS-PAS-HIG-16-027 (2016).

[29] M. Maniatis, Int. J. Mod. Phys. A 25 (2010) 3505 [arXiv:0906.0777 [hep-ph]]. 